\title{
Demonstration of properties of a suppressor variable
}

\author{
DAVID J. KRUS and SUSAN M. WILKINSON \\ Arizona State University, Tempe, Arizona
}

\begin{abstract}
A general logical model of properties of suppressor variables is proposed. Consistent exploration of possible manifestations of suppressor variables within this theoretical framework accounts for extant classifications of suppressor variables into the classical, net, and cooperative categories and suggests existence of new subcategories, not detected previously. The discussed model leads to consistent identification and classification of suppressor variables and facilitates computer simulation.
\end{abstract}

Since Horst (1941) introduced the concept of the suppressor variable, this "quasiparadoxical curiosity" (to use Cohen \& Cohen's, 1975, term) has received sustained attention (Darlington, 1968; Dayton, 1972; Lubin, 1957). In its classical rendering, a suppressor variable has a zero correlation with the criterion, but nevertheless contributes to the predictive validity of a test battery. The current definition of a suppressor variable is that it is a variable which increases regression weights and, thus, increases the predictive validity of other variables in a regression equation (Conger, 1974, pp. 36-37).

Classification of suppressor variables into several categories was a logical outcome of the increase in generality of the suppressor variable concept. Conger (1974) identified three kinds of suppressor variables: traditional, negative, and reciprocal. Cohen and Cohen (1975, pp. 8491) named the same categories classical, net, and cooperative. Succinctly defined,

If the [predictor variable] in question has a zero (in practice very small) correlation with the [criterion variable], the situation is one of classical suppression. If its beta weight is of opposite sign from its [correlation with the criterion], it is serving as a net suppressor. If its beta weight exceeds its correlation with the criterion and is of the same sign, cooperative suppression is indicated. (Cohen \& Cohen, 1975, p. 91)

The same authors also call attention to the fact that a beta coefficient, which falls outside the limits defined by the correlation of its corresponding variable with the criterion and zero, signals the presence of a suppressor variable.

To gain insight into the logical relationships underlying the seemingly paradoxical behavior of suppressor variables, one might well consult the McNemar (1969, pp. 210-211) model. Suppose a predictor variable $\left(X_{1}\right)$ consists of three elements: a second predictor variable $\left(X_{2}\right)$

\footnotetext{
We thank two anonymous reviewers for their help in improving the manuscript. Address correspondence to D. J. Krus, University Testing Services, 302 Payne Hall, Arizona State University, Tempe, AZ 85287.
}

of one element and a criterion variable $(Y)$ of each of two elements. Furthermore, suppose that $X_{1}$ and $X_{2}$ have one element in common, $X_{1}$ and $Y$ have two elements in common, and $X_{2}$ and $Y$ have no overlapping elements:

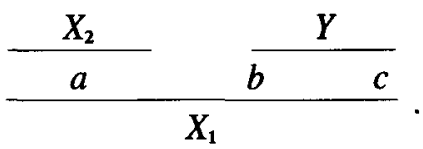

Diagrammatically, the variables and elements represent a suppressor model; however, a special formula for the coefficient of correlation must be used to demonstrate its properties.

In this formula, the correlation $r_{x y}$ is computed as

$$
r_{x y}=n_{c} /\left[\left(n_{x}+n_{c}\right)^{1 / 2}\left(n_{y}+n_{c}\right)^{1 / 2}\right],
$$

where $n_{x}$ equals the number of elements unique to $X, n_{y}$ the number of elements unique to $Y$, and $n_{\mathrm{c}}$ the number of elements common to both variables. For example,

$$
\begin{aligned}
r x_{1} y & =2 /\left[(3+2)^{1 / 2}(2+2)^{1 / 2}\right]=.45, \\
r x_{1} x_{2} & =1 /\left[(3+1)^{1 / 2}(1+1)^{1 / 2}\right]=.35, \text { and } \\
r x_{2} y & =0 /\left[(1+0)^{1 / 2}(2+0)^{1 / 2}\right]=.00 .
\end{aligned}
$$

These correlations represent the prediction model which contains a suppressor variable. However, the necessity to use a special formula to discuss its assumptions limits its utility.

The purpose of this paper is to elaborate and generalize the McNemar model. Also, consistent unfolding of the new model was attempted to demonstrate its ability to account for extant categories of suppressor variables and to indicate whether existence of alternate or additional classification categories is a theoretical possibility.

\section{A GENERAL LOGICAL MODEL OF SUPPRESSOR VARIABLES}

Consider a set of attributes $a_{1}, a_{2}, a_{3}, \ldots, a_{n}$ contained by a set of predictor variables $X_{1}, X_{2}, X_{3}, \ldots, X_{k}$ and by a criterion variable $Y$. Let us restrict the discussion 
to a case in which an attribute is either present (1) or absent $(0)$.

An exhaustive pattern of all possible configurations of the attributes contained by variables in a regression equation will be $2^{k}$. Assume that we could identify all attributes contained by each predictor variable and by the criterion. The response patterns then could serve as a template for construction of a hypothetical data matrix, containing the minimal, but exhaustive, set of response patterns as if all individuals with all possible attributive configurations were included in the analysis. To make this general model suitable for demonstration of properties of suppressor variables, we need to introduce proper constraints. Suppose, that for a minimal regression model of two predictors and a criterion, the predictor variable $X_{1}$ contains $u$ attributes, the predictor variable $X_{2}$ is composed of $v$ attributes, and the criterion variable $Y$ has $w$ attributes. The suppressor variable constraint then can be imposed on the general model so that if $v$ is a subset of $u$ and $w$ is a subset of both $u$ and $v$, then the resulting data set will exhibit properties of a regression model containing a suppressor variable.

For example, consider a case of a hypothetical test, consisting of two predictor variables, $X_{1}$ and $X_{2}$, and a criterion variable $Y$, used for selection of applicants for a position that requires knowledge of addition and multiplication. It may be that one of the arithmetic problems used to assess these abilities contains a problem requiring the knowledge of division for its correct solution. The attributes present in this test battery thus are the abilities to add, multiply, and divide, which may be symbolized as $a_{1}, a_{2}$, and $a_{3}$. A test item, say $X_{1}$, containing all three attributes may be written as

$$
[(3+5) / 7] 8=?
$$

A test item, say $X_{2}$, requiring the knowledge of division may be written as

$$
9 / 3=\text { ? }
$$

The criterion performance $Y$ could be assessed by checking a column of on-the-job additions and multiplications, such as

$$
3(3+7+9+2+8+22+1)=?
$$

Because three attributes are under scrutiny, the matrix of all possible response patterns, assuming (unrealistically) that the independence of the abilities measured will have $2^{3}$ response patterns. Conveniently, this matrix may be constructed by successively halving the necessary alternations of the strings of ones and zeros. Thus, for our example, the total number of eight response patterns is halved into two strings of four ones and four zeros. The resulting pattern is subsequently halved into a string of alternating two ones and two zeros. Finally, a string of alternating ones and zeros is constructed for the third variable. Thus: $a_{1}=[1,1,1,1,0,0,0,0], \quad a_{2}=$ $[1,1,0,0,1,1,0,0$,$] , and a_{3}=[1,0,1,0,1,0,1,0]$. In matrix form all possible response patterns for true abilities under consideration will be

\begin{tabular}{ccc}
$a_{1}$ & $a_{2}$ & $a_{3}$ \\
\hline 1 & 1 & 1 \\
1 & 1 & 0 \\
1 & 0 & 1 \\
1 & 0 & 0 \\
0 & 1 & 1 \\
0 & 1 & 0 \\
0 & 0 & 1 \\
0 & 0 & 0.
\end{tabular}

Because the successful performance on the variable $X_{1}$ necessitates the knowledge of addition, multiplication, and division, only an individual with all three abilities will respond correctly (excluding guesses). Thus, $X_{1}=$ $[1,0,0,0,0,0,0,0]$ for a hypothetical set of 8 individuals, unique with respect to configuration of attributes measured. The second predictor variable $X_{2}$ is a question solvable by dividing the numbers used. Its response vector will therefore mimic the $a_{3}$ vector of true abilities to divide, as $X_{2}=[1,0,1,0,1,0,1,0]$. Finally, the criterion performance $Y$, based on abilities to add and multiply, will be correct only if both the $a_{1}$ and $a_{2}$ abilities are present. Thus, $Y=[1,1,0,0,0,0,0,0]$. In matrix form, the test performance data matrix is

\begin{tabular}{ccc}
$X_{1}$ & $X_{2}$ & $Y$ \\
\hline 1 & 1 & 1 \\
0 & 0 & 1 \\
0 & 1 & 0 \\
0 & 0 & 0 \\
0 & 1 & 0 \\
0 & 0 & 0 \\
0 & 1 & 0 \\
0 & 0 & 0.
\end{tabular}

Its corresponding correlation matrix is

\begin{tabular}{lrrr} 
& $X_{1}$ & $X_{2}$ & \multicolumn{1}{c}{$Y$} \\
\cline { 2 - 4 }$X_{1}$ & 1.00 & .38 & .65 \\
$X_{2}$ & & 1.00 & .00 \\
$Y$ & & & 1.00 \\
\cline { 3 - 4 }
\end{tabular}

This matrix is in accordance with a configuration of correlations necessary to identify a classical suppressor variable.

\section{CATEGORIES OF SUPPRESSOR VARIABLES}

A minimal configuration of the discussed model requires a regression of two predictors, one containing at least two attributes on a criterion having a singular attribute. Suppose $X_{1}$ contains two attributes, $X_{2}$ contains one attribute which is one of the attributes present in variable $X_{1}$, and the criterion variable $Y$ contains a single at- 
tribute which is also present in the variable $X_{1}$, but not in the variable $X_{2}$. The template for these attributes may be constructed as

\begin{tabular}{cc}
$a_{1}$ & $a_{2}$ \\
\hline 1 & 1 \\
1 & 0 \\
0 & 1 \\
0 & 0
\end{tabular}.

The template's corresponding data matrix is presented in the upper left corner of Table 1 . To facilitate the understanding of how this data matrix was constructed from the above template, assume that the variable $X_{1}$ consisted

Table

Logical Model of Suppressor Variables

\begin{tabular}{|c|c|c|c|c|c|c|c|c|c|c|}
\hline & & & & $\mathrm{Neg}$ & ive $\mathrm{C}$ & ssical & Suppre & & & \\
\hline & $X_{1}$ & $X_{2}$ & & & $X_{1}$ & $X_{2}$ & $Y$ & $\underline{r_{i y}^{2}}$ & $\begin{array}{c}\text { Beta } \\
\text { weights }\end{array}$ & $\underline{R}^{2}$ \\
\hline $\begin{array}{l}A \\
B\end{array}$ & $\begin{array}{l}1 \\
0\end{array}$ & $\begin{array}{l}1 \\
0\end{array}$ & $\begin{array}{l}1 \\
1\end{array}$ & $X_{1}$ & 1.00 & .58 & .58 & .33 & .87 & .50 \\
\hline $\begin{array}{l}C \\
D\end{array}$ & $\begin{array}{l}0 \\
0\end{array}$ & $\begin{array}{l}1 \\
0\end{array}$ & $\begin{array}{l}0 \\
0\end{array}$ & $X_{2}$ & & 1.00 & .00 & .00 & -.50 & \\
\hline & & & & & gative & Net Su & ppressi & & & \\
\hline & $X_{1}$ & $X_{2}$ & $Y$ & & $X_{1}$ & $X_{2}$ & $Y$ & $\underline{r_{i y}^{2}}$ & $\begin{array}{c}\begin{array}{c}\text { Beta } \\
\text { weights }\end{array} \\
\end{array}$ & $\underline{R}^{2}$ \\
\hline$A_{1}$ & 1 & 1 & 1 & & & & & & & \\
\hline $\begin{array}{l}A_{2} \\
B\end{array}$ & $\begin{array}{l}1 \\
0\end{array}$ & $\begin{array}{l}1 \\
0\end{array}$ & $\begin{array}{l}1 \\
1\end{array}$ & $X_{1}$ & 1.00 & .67 & .67 & .44 & 1.00 & .58 \\
\hline$C$ & 0 & 1 & 0 & $X_{2}$ & & 1.00 & .17 & .03 & -.50 & \\
\hline$D$ & 0 & 0 & 0 & & & & & - & & \\
\hline & & & & Jegat & $\mathrm{CO}$ & perativ & Supp & sion & & \\
\hline & $X_{1}$ & $X_{2}$ & $Y$ & & $X_{1}$ & $X_{2}$ & $Y$ & $r_{i y}^{2}$ & $\begin{array}{c}\text { Beta } \\
\text { weights }\end{array}$ & $R^{2}$ \\
\hline$A$ & 1 & 1 & 1 & & & & & & & \\
\hline$B_{1}$ & 0 & 0 & 1 & $X_{1}$ & 1.00 & .61 & .41 & .17 & .82 & .44 \\
\hline$B_{2}$ & 0 & 0 & 1 & & & & & & & \\
\hline$C$ & 0 & 1 & 0 & $X_{2}$ & & 1.00 & -.17 & .03 & -.67 & \\
\hline$D$ & 0 & 0 & 0 & & & & & & & \\
\hline & & & & Jegat & $\mathrm{Coc}$ & perativ & Supp & sion & & \\
\hline & $X_{1}$ & $X_{2}$ & $Y$ & & $X_{1}$ & $X_{2}$ & $Y$ & $r_{i y}^{2}$ & $\begin{array}{c}\text { Beta } \\
\text { weights }\end{array}$ & $R^{2}$ \\
\hline$A$ & 1 & 1 & 1 & & & & & & & \\
\hline$B$ & 0 & 0 & 1 & $X_{1}$ & 1.00 & .41 & .61 & .38 & .82 & .58 \\
\hline$C_{1}$ & 0 & 1 & 0 & & & & & & & \\
\hline$C_{2}$ & 0 & 1 & 0 & $X_{2}$ & & 1.00 & -.17 & .03 & -.50 & \\
\hline$D$ & 0 & 0 & 0 & & & & & & & \\
\hline & & & & & gative & Net $\mathrm{Su}$ & ppressi & & & \\
\hline & $X_{1}$ & $X_{2}$ & $Y$ & & $X_{1}$ & $X_{2}$ & $Y$ & $\underline{r_{i y}^{2}}$ & $\begin{array}{c}\begin{array}{c}\text { Beta } \\
\text { weights }\end{array} \\
\end{array}$ & $\underline{R^{2}}$ \\
\hline$A$ & 1 & 1 & 1 & & & & & & & \\
\hline$B$ & 0 & 0 & 1 & $X_{1}$ & 1.00 & .61 & .61 & .38 & .82 & .44 \\
\hline$C$ & 0 & 1 & 0 & & & & & & & \\
\hline$D_{1}$ & 0 & 0 & 0 & $X_{2}$ & & 1.00 & .17 & .03 & -.33 & \\
\hline$D_{2}$ & 0 & 0 & 0 & & & & & - & & \\
\hline
\end{tabular}

Table 2

Logical Model of Suppressor Variables with Reflected Suppressor Variable $X_{2}$

Positive Classical Suppression

$$
\begin{array}{cccc} 
& X_{1} & X_{2} & Y \\
\cline { 2 - 4 } A & 1 & 0 & 1 \\
B & 0 & 1 & 1 \\
C & 0 & 0 & 0 \\
D & 0 & 1 & 0 \\
\hline
\end{array}
$$

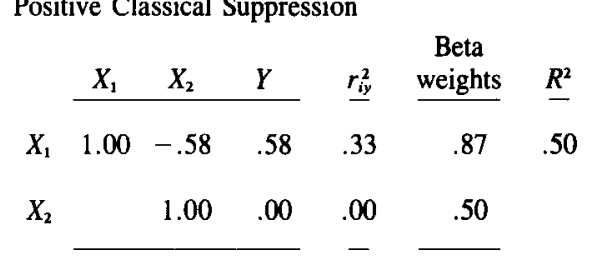

Positive Net Suppression

$$
\begin{array}{lccc} 
& X_{1} & X_{2} & Y \\
\cline { 2 - 4 } A_{1} & 1 & 0 & 1 \\
A_{2} & 1 & 0 & 1 \\
B & 0 & 1 & 1 \\
C & 0 & 0 & 0 \\
D & 0 & 1 & 0 \\
\hline
\end{array}
$$

$$
\begin{aligned}
& \begin{array}{llllll}
X_{1} & X_{2} & Y & \underline{r_{i y}^{2}} & \begin{array}{c}
\text { Beta } \\
\text { weights }
\end{array} & \underline{R^{2}}
\end{array} \\
& \begin{array}{lllllll}
X_{1} & 1.00 & -.67 & .67 & .44 & 1.00 & .58
\end{array} \\
& \begin{array}{lllll}
X_{2} & 1.00 & -.17 & .03 & .50
\end{array}
\end{aligned}
$$

Positive Cooperative Suppression

\begin{tabular}{lccc} 
& $X_{1}$ & $X_{2}$ & $Y$ \\
\cline { 2 - 4 }$A$ & 1 & 0 & 1 \\
$B_{1}$ & 0 & 1 & 1 \\
$B_{2}$ & 0 & 1 & 1 \\
$C$ & 0 & 0 & 0 \\
$D$ & 0 & 1 & 0
\end{tabular}

$$
\begin{aligned}
& \begin{array}{llllll}
X_{1} & X_{2} & Y & r_{i y}^{2} & \underline{\text { weights }} & R^{2}
\end{array} \\
& \begin{array}{llllllll}
X_{1} & 1.00 & -.61 & .41 & .17 & .82 & .44
\end{array} \\
& \begin{array}{lllll}
X_{2} & 1.00 & .17 & .03 & .67
\end{array}
\end{aligned}
$$

Positive Cooperative Suppression

\begin{tabular}{lccc} 
& $X_{1}$ & $X_{2}$ & $Y$ \\
\cline { 2 - 4 }$A$ & 1 & 0 & 1 \\
$B$ & 0 & 1 & 1 \\
$C_{1}$ & 0 & 0 & 0 \\
$C_{2}$ & 0 & 0 & 0 \\
$D$ & 0 & 1 & 0 \\
\hline
\end{tabular}

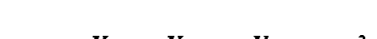

Beta

weights $\quad \underline{R^{2}}$

$\begin{array}{llllllll}X_{1} & 1.00 & -.41 & .61 & .38 & .82 & .58\end{array}$

$\begin{array}{lllll}X_{2} & 1.00 & .17 & .03 & .50\end{array}$

Positive Net Suppression

\begin{tabular}{lccc} 
& $X_{1}$ & $X_{2}$ & $Y$ \\
\cline { 2 - 4 }$A$ & 1 & 0 & 1 \\
$B$ & 0 & 1 & 1 \\
$C$ & 0 & 0 & 0 \\
$D_{1}$ & 0 & 1 & 0 \\
$D_{2}$ & 0 & 1 & 0 \\
\hline
\end{tabular}

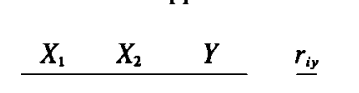

Beta

weights $\quad R^{2}$

$\begin{array}{lllllll}X_{1} & 1.00 & -.61 & .61 & .38 & .82 & .44\end{array}$

$\begin{array}{lllll}X_{2} & 1.00 & -.17 & .03 & .33\end{array}$

of a single question, testing for an ability to add and subtract; for example

$$
2+3-1=?
$$

The variable $X_{2}$ consisted of single-item testing for an ability to subtract; for example

$$
2-3=\text { ? }
$$

The criterion variable $Y$ tested for addition; for example

$$
3+7=\text { ? }
$$

Using the above template, the first row of the data matrix was written as a string of ones because the first hypothet- 
Table 3

Classification Based on the Logical Model of Suppressor Variables

\begin{tabular}{|c|c|c|}
\hline $\begin{array}{c}\text { Correlation } \\
\text { with Criterion }\end{array}$ & $\begin{array}{c}\text { Beta } \\
\text { Weight }\end{array}$ & Nomenclature \\
\hline zero & negative & Negative classical suppression \\
\hline zero & positive & Positive classical suppression \\
\hline positive & positive & Positive cooperative suppression \\
\hline positive & negative & Negative net suppression \\
\hline negative & positive & Positive net suppression \\
\hline negative & negative & Negative cooperative suppression \\
\hline
\end{tabular}

ical subject possessed all skills necessary to solve the problems. The digit in the second row and first column is zero, because two abilities are necessary to solve the first problem, and the second subject possesses only one. The next zero indicates that even though only a single ability is necessary, the subject, knowing how to add but not how to subtract, will not pass the item. The last number, a one, indicates that the second subject knows how to add. The remaining two lines were formed in the same fashion.

The matrix of intercorrelations, adjacent to the first data matrix in Table 1, together with its corresponding vector of beta weights indicates that the variable $X_{2}$ is a classical suppressor variable.

In the case of real data matrices, the presence of exactly equal proportions of all four possible response patterns is unlikely. This situation was simulated in the second data matrix in Table 1, where the first theoretical response pattern $A$ was included twice. The resulting matrix of intercorrelations displayed the properties of a net suppression. The remaining three data matrices were simulated by successively doubling the remaining response patterns. Their corresponding correlation matrices and vectors of beta weights displayed variations of characteristics of the cooperative and net suppression classification categories.

Reflection of a variable reverses its orientation, but does not disturb the latent structure of the data set. Within the framework of the present model, it appeared plausible to reflect the suppressor variable $X_{2}$. Results of this operation are depicted in Table 2 . The regression solution for this data matrix can again be classified as a classical suppression, but of a different type.

The remaining data matrices in Table 2 simulate the variations of over- and underrepresentations of successive types of response patterns. The corresponding regression solutions identify the categories of net and cooperative suppressions and its corresponding subcategories.

Comparing patterns of negative-zero-positive correlations of suppressor variables with the criterion and their corresponding beta weights, six distinctive patterns emerged, as shown in Table 3. The generic categories of the suppressor variables appear to be the cases of negative and positive suppression. Subcategories of negative net and cooperative suppressors are derived from the generic category of the negative classical suppression. Subcategories of positive net and cooperative suppression are derived from the generic category of the positive classical suppression.

\section{DISCUSSION}

The suggested logical model of properties of suppressor variables offers a method for systematic construction of idealized data matrices displaying configurations of intercorrelations, predictor-criterion correlations, beta weights, and resulting multiple regression solutions relevant for description of categories of suppressor variables.

The proposed classification scheme naturally unfolds from the postulates of the extended McNemar (1969) model. It is concise and closed within the framework of the mutually exclusive and exhausitve categories delineated at the inception of our discussion.

The explanatory potential of the described theoretical model is substantial. It provides means for consistent identification of suppressor variables and their precise classifications. It also lends itself naturally to computer simulation.

\section{AVAILABILITY}

The program allowing classroom demonstration of the discussed properties of suppressor variables was written for computers using Intel's iAPX 88/186/286 series of microprocessors (IBM AT/XT/PC, TANDY $1000 / 2000 / 3000$, and many others). For a free copy, please send a formatted (two sides, 40 tracks/side, nine sectors/track, 512 bytes/sector) blank diskette with selfaddressed, return protective wrapping to D. J. Krus, University Testing Services, 302 Payne Hall, Arizona State University, Tempe, AZ 85287.

\section{REFERENCES}

Conger, A. J. (1974). A revised definition for suppressor variables: A guide to their identification and interpretation. Educational \& Psychological Measurement, 34, 35-46.

COHEN, J., \& COHEN, P. (1975). Applied multiple regression/correlation analysis for the behavioral sciences. New York: Wiley.

Dartington, R. B. (1968). Multiple regression in psychological research and practice. Psychological Bulletin, 69, 161-182.

Dayton, C. M. (1972). A method for constructing data which illustrate a suppressor variable. The American Statistician, 26(5), 36.

Horst, P. (1941). The role of predictor variables which are independent of the criterion. Social Science Research Bulletin, 48, 431-436.

LubiN, A. (1957). Some formulae for use with suppressor variables. Educational \& Psychological Measurement, 17, 286-296.

McNemar, Q. (1969). Psychological statistics. New York: Wiley.

(Manuscript received May 8, 1985; revision accepted for publication February 18, 1986.) 